\title{
Deciphering Deconfinement in Correlations of Conserved Charges
}

\author{
A. Majumder \\ Department of Physics, Duke University, Durham, NC 27708-0305, USA
}

\begin{abstract}
Diagonal and off-diagonal flavor and conserved charge susceptibilities reveal the prevalent degrees of freedom of heated strongly interacting matter. Results obtained from lattice simulations are compared with various model estimates in an effort to weed down various possible pictures of a quark gluon plasma. We argue that the vanishing of the off-diagonal quark flavor susceptibilities and its derivatives with respect to chemical potential, at temperatures above $1.5 \mathrm{Tc}$, can only be understood in a picture of a gas or liquid composed of quasi-particles which carry the same quantum numbers as quarks and antiquarks. A potential new observable, blind to neutral and nonstrange particles, is introduced and related via isospin symmetry to the ratio of susceptibilities of baryonic strangeness to strangeness generated in the excited matter created at RHIC.
\end{abstract}

PACS numbers: 12.38.Mh, 11.10.Wx, 25.75.Dw

The goal of of the heavy-ion program at the Relativistic Heavy Ion Collider (RHIC) is the creation and study of heated strongly interacting matter at nearly vanishing baryon density $[1,2]$. The experimental results of the four RHIC detector collaborations, have set a lower bound of about $5 \mathrm{GeV} / \mathrm{fm}^{3}$ on the energy density at a time $\tau=1 \mathrm{fm} / \mathrm{c}$ in central $\mathrm{Au}+\mathrm{Au}$ collisions [3]. Such an energy density should place the produced matter well into region of the QCD phase diagram that cannot be described as a hadronic resonance gas. Matter in this domain of the QCD phase diagram had been expected to be a colored plasma composed of quasiparticle excitations with the quantum numbers of quarks and gluons [1,4]. The produced matter, however, clearly exhibits collective behavior as evidenced by its radial and elliptic flow [5]. This result suggests that there must exist a strong interaction between the constituents of the medium.

Lattice simulations of QCD at finite temperature and vanishing chemical potential have demonstrated the existence of a phase transition at $T=T_{c} \simeq 170 \mathrm{MeV}$ signalled by a steep rise in the energy density and the pressure as a function of the temperature. The slow rise of both quantities prior to the sudden transition has been successfully modelled by a picture of a hadronic resonance gas [6]. A similar program for the description of the excited phase as a weakly interacting plasma of quasiparticles [7] has not met with success in the region from $T_{c} \leq T \leq 3 T_{c}$. This indicates that matter in this region may not be a weakly coupled plasma where quarks and gluons are deconfined over large distances. It is clear that a microscopic understanding of the emergent degrees of freedom in this regime is essential in order to understand the matter created in nuclear collisions at RHIC. The object of these proceedings is to outline a set of flavor off-diagonal susceptibilities which may be calculated 
on the lattice, in phenomenological models and in certain situations may even be measured in experiments. Such off-diagonal susceptibilities are discerning probes of the thermodynamic degrees of freedom in strongly interacting QCD matter, both above and below $T_{c}$.

In the lattice formulation of $\mathrm{QCD}$, the fundamental degrees of freedom are local quark and gluon fields. Under conditions where deconfinement has been achieved, the elementary set of conserved charges is given by the net contents of each quark flavor $u, d, s$. An alternate basis is provided by the hadronically defined conserved charges of baryon number $(B)$, electric charge $(Q)$ and strangeness $(S)$. The (co-)variances between any two conserved charges $(x, y)$ are extensive quantities: $\sigma_{x y}^{2}=V T \chi_{x y}$, where $\chi_{x y}$ is the intensive diagonal or off-diagonal susceptibility. These susceptibilities can be measured on the lattice. In heavy-ion experiments, the variances and covariances are measured by means of an event-by-event analysis of the corresponding conserved quantities, i.e.,

$$
\sigma_{x y}^{2}=\frac{1}{N_{E}} \sum_{i \in E} X_{i} Y_{i}-\left(\frac{\sum_{i \in E} X_{i}}{N_{E}}\right)\left(\frac{\sum_{i \in E} Y_{i}}{N_{E}}\right)
$$

where $E$ represents the set of events, $N_{E}$ is the number of events considered, and $X_{i}, Y_{i}$ are the net values of the conserved charge in a given event $i$. The volume independent ratios of variances, measured event-by-event in heavy-ion collisions, may then be directly compared with the lattice estimate for the ratio of susceptibilities.

In the case that the degrees of freedom or active flavors $f$ are eigenstates of the quantum numbers $x, y$, the above expression may be decomposed using $X_{i}=\sum_{f} n_{i}^{f} x_{f}$ where $n_{i}^{f}$ are the number of states of flavor $f$ in event $i$. For independent flavors, where $\left\langle n^{f} n^{g}\right\rangle=\sum_{i \in E} n_{i}^{f} n_{i}^{g}=\left\langle n^{f}\right\rangle\left\langle n^{g}\right\rangle$, one may easily demonstrate that (see Ref. [8] for details)

$$
\sigma_{x y}^{2}=\sum_{f} \sigma_{f}^{2} x_{f} y_{f} \stackrel{P . S}{=} \sum_{f}\left\langle n^{f}\right\rangle x_{f} y_{f} .
$$

The last equality in the above equation holds solely in the case that Poisson statistics is applicable to the independent flavors (i.e., the variance $\sigma^{2}$ is equal to the mean $\langle n\rangle$ ) Equation (2) clearly demonstrates the sensitivity of the off-diagonal susceptibilities to the presence of flavors carrying more than one quantum number, and may be used to estimate the susceptibilities in various microscopic models.

A robust set of ratios of susceptibilities, which has been measured on the lattice and demonstrates direct sensitivity to the flavor carrying degrees of freedom are the two coefficients (see Refs. $[9,10]) C_{B S}=-3 \sigma_{B S}^{2} / \sigma_{S}^{2}$ and $C_{Q S}=3 \sigma_{Q S}^{2} / \sigma_{S}^{2}$. The susceptibilities may be computed phenomenologically, assuming the condition of Eqs. (2). Plotted in the left panel of Fig. 1. are the coefficients $C_{B S}$ and $C_{Q S}$ as obtained from a hadronic resonance gas spectrum, truncated at the mass of the $\Omega^{-}$ along with the results obtained from the lattice. One notes, that the hadron resonance gas provides a good description of the behavior of the ratio of susceptibilities up to the point of the phase transition. Here the behavior of the truncated spectrum fails to reproduce the sharp rise in $C_{B S}$ and the corresponding sharp drop in $C_{Q S}$.

The results from the lattice simplify in the high temperature phase, where one notes the following simple relations [11, 12];

$$
\chi_{u s}=\chi_{d s} \leq \chi_{u d} \ll \chi_{s} \leq \chi_{d}=\chi_{u},
$$



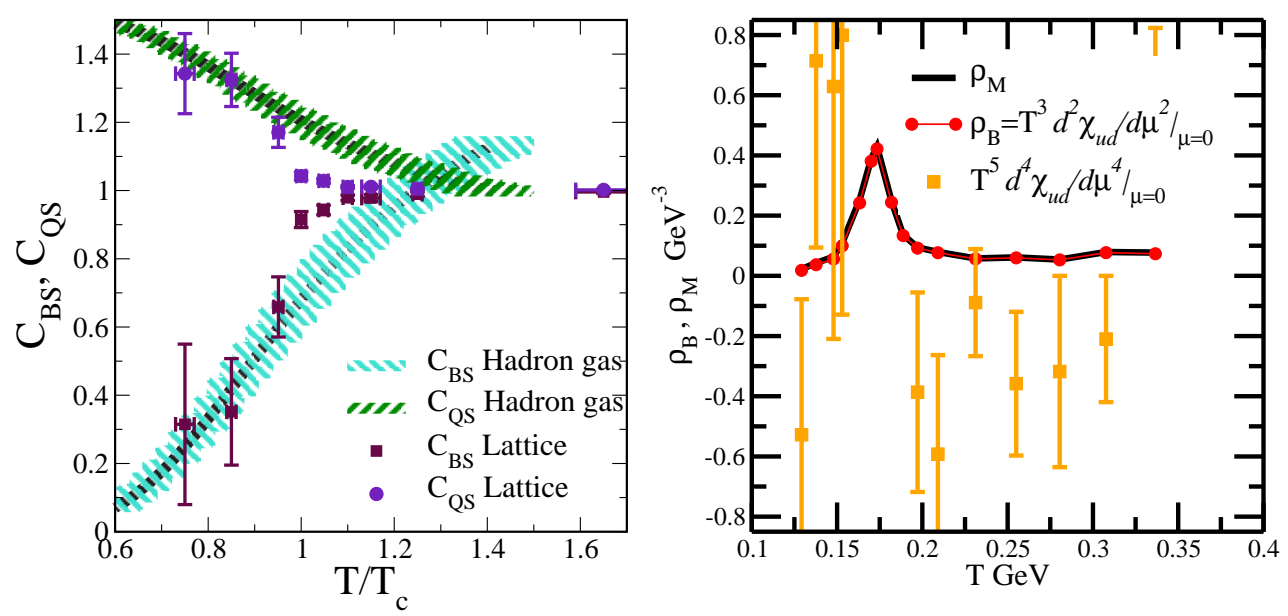

Figure 1. (Left Panel) A comparison of $C_{B S}, C_{Q S}$ calculated in a truncated hadron resonance gas at $\mu_{B}=\mu_{S}=\mu_{Q}=0 \mathrm{MeV}$ compared to lattice calculations from Ref. [10]. The two hazed bands for $C_{B S}$ and $C_{Q S}$ for the hadron gas plots reflect the uncertainty in the actual value of the phase transition temperature $T_{c}=170 \pm 10 \mathrm{MeV}$.

(Right Panel) A plot of the mesonic and baryonic contributions to $\chi_{u d}(T, \mu=0)$ and and a plot of the derivatives $T \partial^{2} \chi_{u d} /\left.\partial(\mu \beta)^{2}\right|_{\mu=0}$ (which is equal to the baryonic contribution $\rho_{B}$ ) and $T \partial^{4} \chi_{u d} /\left.\partial(\mu \beta)^{4}\right|_{\mu=0}$ (which is negative beyond $T_{c}=170 \mathrm{MeV}$ and thus is inconsistent with a bound state interpretation).

where the last and the first equality applies in the case of isospin symmetry. For simulations at vanishing chemical potentials $\langle B\rangle=\langle S\rangle=\langle Q\rangle=0$, as a result, we obtain,

$$
C_{B S}=-3 \frac{\langle B S\rangle}{\left\langle S^{2}\right\rangle}=\frac{\langle(u+d+s) s\rangle}{\left\langle s^{2}\right\rangle} \approx \frac{\chi_{u s}+\chi_{d s}+\chi_{s}}{\chi_{s}} \approx 1 .
$$

Alternatively, one may state that $\chi_{u s} \simeq \chi_{d s} \rightarrow 0$. Models of the deconfined phase must obey such constraints. The simplest model of deconfined matter is that of non-interacting quark, anti-quark and gluon quasi-particles. In such a situation, offdiagonal susceptibilities are identically zero as the degrees of freedom may carry only a single flavor.

Recently, an alternate picture of the deconfined phase has been proposed, where a tower of bound states of quarks and gluons are present besides the quasiparticles themselves [13]. The large cross-sections from resonance scattering imply short mean free paths and lend consistency to the macroscopic hydrodynamic picture henceforth used to describe the dynamical evolution of the matter produced at RHIC. However, in a plasma containing bound states of quarks, in the form of colored mesons, diquarks and quark-gluon bound states, the correlation between flavors is no longer negligible. In a gas consisting solely of quark-antiquark bound states, built from three flavors of quarks, the contributing states are $u \bar{d}, d \bar{u}, u \bar{s}, s \bar{u}, d \bar{s}, s \bar{d}$ (The flavor singlets $q \bar{q}$ and gluon bound states will be ignored as they carry no conserved flavor). In such a system, the ratio $C_{B S}$ must vanish, because all states have vanishing baryon number. Thus meson like states produce a considerable negative contribution to the off-diagonal susceptibilities $\chi_{u s}, \chi_{u d}, \chi_{d s}$ and tend to depress the value of $C_{B S}$ from unity. Quark gluon bound states contribute similarly as quark-antiquark quasiparticles. Inclusion 
of all such states at a temperature of $T=1.5 T_{c}$ yields a value $C_{B S}=0.62$ (or equivalently, negative off-diagonal susceptibilities) quite different from the values found on the lattice [9].

These results have motivated the inclusion of a variety of baryonic states into the model outlined above [14]. Baryonic states provide additive contributions to the off-diagonal susceptibilities and as a result also to $C_{B S}$. The inclusion of a sufficient number of baryonic states may lead to a balance between the mesonic and baryonic contributions and one may engineer a $\chi_{u s}=\chi_{d s}=0$ and as a result a $C_{B S}=1$ in agreement with the lattice. To discern between these two possibilities, one turns to higher derivatives of the the off-diagonal susceptibilities in terms of the baryonchemical potential $\mu_{B}$.

In the interest of simplicity, the flavor group will be restricted to $\mathrm{SU}(2)_{f}$. Lattice results for susceptibilities and their derivatives with respect to baryon chemical potential using dynamical quarks exist in this case [12]. The model will consist of quark and anti-quark quasi-particles $u, d$ and $\bar{u}, \bar{d}$, meson like bound states $u \bar{u}, d \bar{d}, u \bar{d}, d \bar{u}$, diquark states $u u, d d, u d$ and their antiparticles as well as baryons uuu, uud, udd, $d d d$ and the corresponding anti-baryons. In this simplified situation, one may derive the following simple relations between the off-diagonal susceptibility and the meson and baryon densities [8] $\left(\rho_{x}^{0}=n_{x}^{0} / V\right.$, where $n_{x}^{0}$ denotes the population of a given flavor at $\mu=0$ and not the difference between the flavor anti-flavor populations)

$$
\begin{aligned}
& \left.T \chi_{u d}(T, \mu)\right|_{\mu=0} \simeq-2 \rho_{u \bar{d}}^{0}(T)+\left\{2 \rho_{u d}^{0}(T)+4 \rho_{u u d}^{0}(T)+4 \rho_{u d d}^{0}(T)\right\}, \\
& T\left[\frac{\partial^{2} \chi_{u d}}{\partial(\mu \beta)^{2}}\right]_{\mu=0}=2 \rho_{u d}^{0}(T)+4 \rho_{u u d}^{0}(T)+4 \rho_{u d d}^{0}(T)=T\left[\frac{\partial^{4} \chi_{u d}}{\partial(\mu \beta)^{4}}\right]_{\mu=0} .
\end{aligned}
$$

In this way, one may divide the contributions to the off-diagonal susceptibility and its derivatives in terms of mesonic $\left(\rho_{M}=2 \rho_{u \bar{d}}^{0}(T)\right)$ and baryonic $\left(\rho_{B}=\right.$ $\left.2 \rho_{u d}^{0}(T)+4 \rho_{u u d}^{0}(T)+4 \rho_{u d d}^{0}(T)\right)$ contributions. Using the measured susceptibility and its derivatives, these densities are estimated as a function of the temperature. and plotted in the right panel of Fig. 1. These densities satisfy Eq. (5) and the first equality of Eq. (6). The second condition imposed by Eq. (6) has to be satisfied by the fourth derivative of the susceptibility in such a picture of bound states. The fourth derivative of the susceptibility, as measured on the lattice, has been plotted as the square symbols. Despite large error bars, one notes that while the baryon density or the second derivative of the susceptibility is consistent with Eq. (6) below the phase transition temperature, it becomes inconsistent with such a condition above the phase transition temperature. Above $T_{c}, T \partial^{4} \chi_{u d} / \partial(\mu \beta)^{4}$ is actually negative; hence, no bound state picture is compatible with such results. It has already been pointed out in Ref. [12] that the signs of the various derivatives of the susceptibility are consistent with the picture of a weakly interacting quasi-particle gas.

In the experimental measurement of baryon-strangeness correlations, any detector has to accurately assess the baryon number and strangeness in a given rapidity bin in each event. As most detectors are blind to stable uncharged particles they cannot measure the neutron and antineutron populations. As a result, a measurement of $\sigma_{B S}$ may become rather difficult. As a recourse, a new quantum number is constructed, $M=B+2 I_{3}$, and fluctuations of $M$ with respect to $S$ are studied. One notes that the assumption of isospin symmetry reduces the covariance $\sigma_{M S}$ to simply $\sigma_{B S}$ (see Ref. [8] for details). As a result, in all theoretical models with isospin symmetry $C_{M S}=C_{B S}$. In the experimental determination, $M$ has the advantage that it is vanishing for all particles that do not carry charge or strangeness, thus $M=0$ for neutrons, 


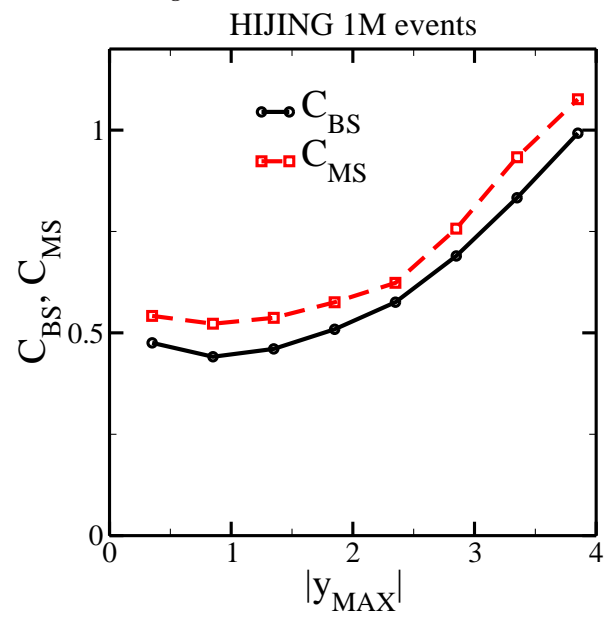

Figure 2. A comparison of the two related ratios of variances $C_{B S}, C_{M S}$ as a function of the acceptance in rapidity from $-\left|y_{\max }\right|$ to $\left|y_{\max }\right|$.

antineutrons, neutral pions, etc.. There exists a greater possibility of contamination of such a signal from charged pions $\left(\pi^{ \pm}\right.$do not contribute to the measurement of $\left.C_{B S}\right)$. The amount of contamination is assessed by comparing measurements of $C_{B S}$ and $C_{M S}$ in an identical set of HIJING events [15].

In Fig. 2 the correlations $C_{B S}$ and $C_{M S}$ are estimated in central $A u-A u$ events at $\sqrt{s}=200 A \mathrm{GeV}$. The acceptance in rapidity ranges from $-\left|y_{\max }\right|$ to $\left|y_{\max }\right|$, hence a larger $y_{\max }$ indicates a larger acceptance. The results are presented as a function of $y_{\max }$. One notes that over the range of $y_{\max }$ the two correlations $C_{B S}$ and $C_{M S}$ are rather similar. This bodes well for the measurement of $C_{B S}$ in RHIC experiments via a measurement of the quantity $C_{M S}$, over a range of rapidity intervals.

In these proceedings, we have demonstrated that the off-diagonal susceptibilities such as $\chi_{u d}, \chi_{u s}, \chi_{d s}$ and the ratio $C_{B S}$ are descerning probes of the flavor structure of heated strongly interacting matter. They may be calculated on the lattice, in models and even measured in RHIC experiments allowing for a systematic exploration of degrees of freedom of deconfined matter.

Work supported by the U.S. Department of Energy, grant DE-FG02-05ER41367.

[1] J. W. Harris and B. Müller, Ann. Rev. Nucl. Part. Sci. 46, 71 (1996).

[2] M. Gyulassy and L. McLerran, Nucl. Phys. A 750, 30 (2005).

[3] I. Arsene et al., Nucl. Phys. A 757, 1 (2005); B. B. Back et al., ibid. 757, 28 (2005); J. Adams et al., ibid. 757, 102 (2005); K. Adcox et al., ibid. 757, 184 (2005).

[4] D. H. Rischke, Prog. Part. Nucl. Phys. 52, 197 (2004). [arXiv:nucl-th/0305030].

[5] K. H. Ackermann et al. Phys. Rev. Lett. 86, 402 (2001); S. S. Adler et al. ibid. 91, 182301 (2003).

[6] F. Karsch, K. Redlich and A. Tawfik, Phys. Lett. B 571, 67 (2003).

[7] J. O. Andersen, E. Braaten and M. Strickland, Phys. Rev. D 61, 074016 (2000); J. P. Blaizot, E. Iancu and A. Rebhan, Phys. Rev. D 68, 025011 (2003).

[8] A. Majumder and B. Muller, arXiv:nucl-th/0605079.

[9] V. Koch, A. Majumder and J. Randrup, Phys. Rev. Lett. 95, 182301 (2005); A. Majumder, V. Koch and J. Randrup, arXiv:nucl-th/0510037.

[10] R. V. Gavai and S. Gupta, arXiv:hep-lat/0510044.

[11] R. V. Gavai and S. Gupta, Phys. Rev. D 67, 034501 (2003); ibid. 65, 094515 (2002).

[12] C. R. Allton et al., Phys. Rev. D 71, 054508 (2005).

[13] E. V. Shuryak and I. Zahed, Phys. Rev. C 70, 021901 (2004); Phys. Rev. D 70, 054507 (2004).

[14] J. Liao and E. V. Shuryak, arXiv:hep-ph/0510110.

[15] X. N. Wang and M. Gyulassy, Phys. Rev. D 44, 3501 (1991). 